\title{
Lymphocytic Interstitial Pneumonia
}

National Cancer Institute

\section{Source}

National Cancer Institute. Lymphocytic Interstitial Pneumonia. NCI Thesaurus. Code C27558.

Interstitial pneumonia characterized by the presence of bibasilar pulmonary interstitial infiltrates composed of lymphocytes and plasma cells. It may be associated with autoimmune and lymphoproliferative disorders. Signs and symptoms include fever, cough, and dyspnea. Symptomatic patients may require immunosuppressive treatment. 